\title{
High-Dynamic Optical Network Control Architecture Based on Spatiotemporal Synchronization
}

\author{
Nan Hua ${ }^{1,2}$, Ruijie Luo ${ }^{1,2}$, Xiaoping Zheng ${ }^{1,2}$ \\ 1. Beijing National Research Center for Information Science and Technology (BNRist), Beijing, \\ 100084, P. R. China \\ 2. Department of Electronic Engineering, Tsinghua University, Beijing, 100084, P. R. China \\ huan@mail.tsinghua.edu.cn
}

\begin{abstract}
We present a novel high-dynamic optical network control architecture based on spatiotemporal synchronization. By introducing high-precision time synchronization, control coordination among nodes and controllers can be established to improve the network dynamic performance by orders of magnitude.
\end{abstract}

Keywords: spatiotemporal synchronization, control coordination, network dynamic performance

\section{INTRODUCTION}

The development of emerging network applications such as data centers [1], high performance computing [2], and 5G mobile communications [3] places higher demands on the dynamic performance of optical network resource allocation. However, at present, the control systems of dynamic optical networks are based on signaling interaction between nodes, or between nodes and controllers, which relies on messages to trigger changing the state of each node to achieve specific network functions [4]. However, due to the existence of signaling delay and processing delay in the traditional network control mechanism, it is difficult to further improve the dynamic performance of optical networks in mechanism. In order to solve this problem, we introduce a new type of high-dynamic optical network control architecture based on spatiotemporal synchronization. By introducing high-precision time synchronization, a control coordination mechanism between nodes and controllers is established in the time dimension, making it possible to improve the dynamic performance of optical networks by orders of magnitude. To achieve this goal, we conducted preliminary explorations and achieved preliminary feasibility results in numerous application scenarios, including but not limited to fine-grained optical data center networks, high-performance computing systems, high-dynamic core/metro optical networks, low-latency metro-access optical networks, and large-scale smart-grid communication networks, laying the foundation for further research of a new generation of high-dynamic spatiotemporal synchronization optical networks.

The rest of the paper is organized as follows. Section 2 explicates the concept of spatiotemporal synchronization optical network control mechanism. Section 3 introduces typical application scenarios of spatiotemporal synchronization optical networks and some preliminary results are also presented in this section. Finally, Section 4 concludes the paper.

\section{SPATIOTEMPORAL SYNCHRONIZATION NETWORK CONTROL MECHANISM}

The control mechanisms of optical networks determine their dynamic performance. In traditional optical networks, the control mechanism is based on signaling interaction between nodes, which relies on messages to trigger every nodal operation, as shown in Fig. 1. Due to the uncertainty of signaling transmission delay, it is difficult to guarantee the arrival time of a signaling packet, and thus network resources with short time windows are not able to be utilized. This restricts the optical networks from improving their dynamic performance, and further leads to a long connection establishment/teardown time at the level of seconds [5]. By using the SDN-based centralized control mechanism, this time can be reduced to several hundred milliseconds [6], but it is difficult to be further improved.

To improve the dynamic performance of optical networks, we introduce a novel spatiotemporal synchronization optical network control mechanism enabled by high-precision time synchronization, which is currently a mature technology [7-9]. In work [7], sub-microsecond time synchronization was achieved in the range of 1014 kilometer. With the help of high-precision time synchronization, nodal operations at different nodes can be executed coordinately.

In the spatiotemporal synchronization control mechanism, the traditional "message-triggered" mode is changed to the "time-triggered" mode as shown in Fig. 1. More specifically, before a serial of nodal operations start, the control packets containing precise operation time labels are sent to the corresponding nodes in advance. Each node then executes the corresponding operations at the exact time in the time labels to coordinately perform the network control functions 
such as connection establishment and teardown. In this way, the decoupling between signaling transmission and nodal operation can be achieved, making it possible to utilize network resources with short time windows to improve the network dynamic performance.

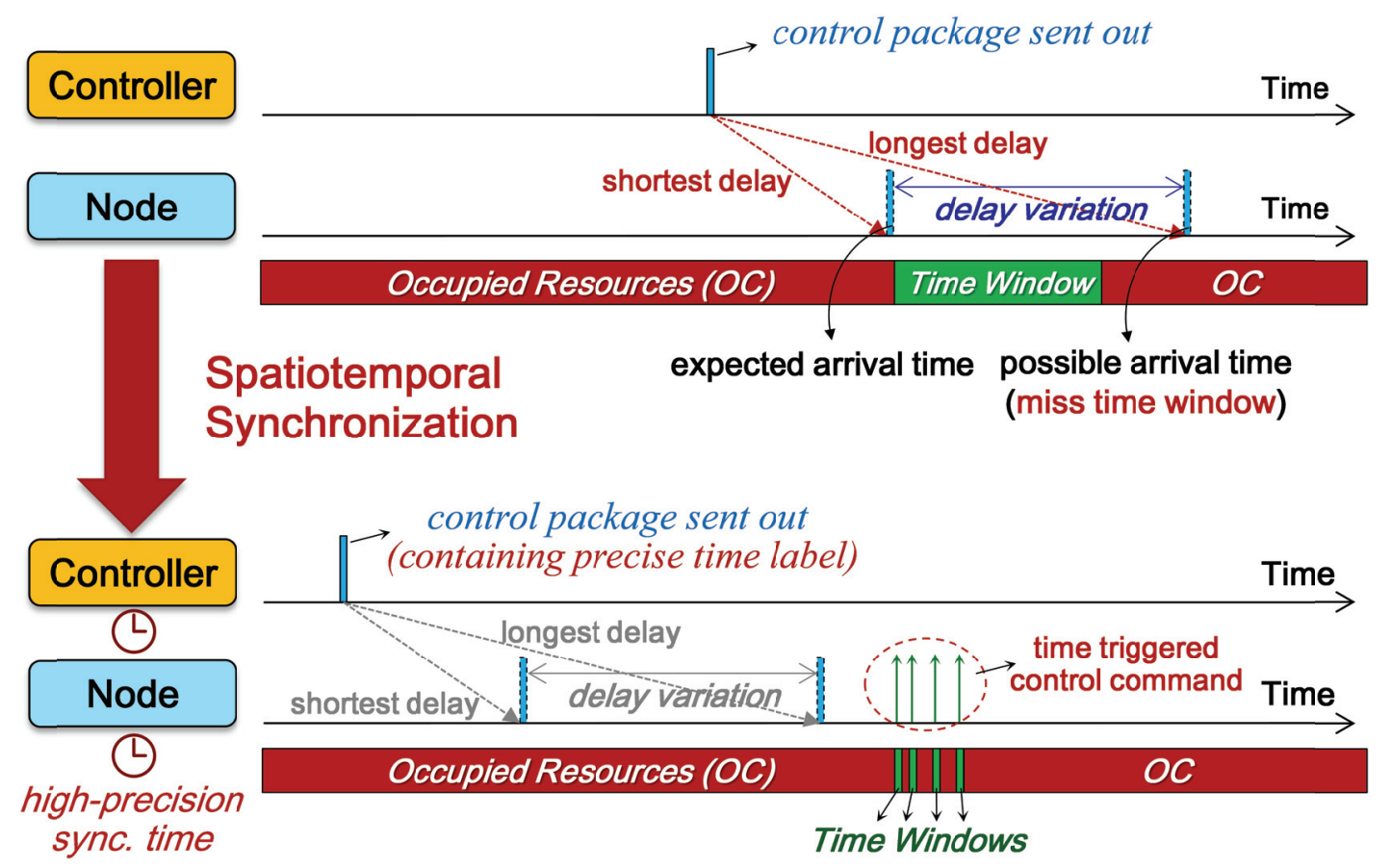

Fig. 1. Optical network control mechanisms comparison: traditional vs. spatiotemporal synchronization

\section{APPLICATION SCENARIOS}

The proposed high-dynamic spatiotemporal synchronization optical network control mechanism can apply to most application scenarios in which network operations can be determined in advance. This section introduces some of the typical application scenarios and presents some of the preliminary results.

\subsection{Fine-grained optical data center networks (DCN)}

Introducing all-optical switching to establish lowlatency optical bypass can provide a solution to the latency problem of DCNs; however, its effect is limited by the restricted switching granularity. To solve this problem, a hybrid packet-OCS intra-DCN architecture was proposed based on the fine-grained bufferless optical time slice switching (OTSS) technology enabled by high-precision time synchronization [10,11]. Prototype experiments validated the feasibility of this architecture which was able to provide sub-wavelength all-optical channel with the granularities of as fine as $1: 100[12]$.

\subsection{High-performance computing (HPC) systems}

Low computational efficiency caused by the mismatch between computing job schedule and communication job schedule is a long-standing problem. To solve this problem, we introduced the synchronized optical network control mechanism into HPC systems, and further proposed the concept of joint allocation of computation and communication resources (JACC). Compared to the traditional methods in which computing and communication resources were allocated separately, JACC could reduce average traffic buffering time by $80 \%$, and realize 50 -ns-level communication time in a prototype experimental system [13].

\subsection{High-dynamic core/metro optical networks}

Traditional "message-triggered" control mechanism becomes a major bottleneck to the network dynamic performance, making it difficult to implement important network functions such as fast network reconfiguration and quick massive network recovery. By introducing the spatiotemporal synchronization control architecture, the "message-triggered" network control mechanism turns into the "time-triggered" one, leading to substantial improvement of the network dynamics. This makes possible the high-dynamic network functions. Experimental and simulation results showed that, in this architecture network reconfiguration time can be reduced by almost three orders of magnitude [14]. In another work, a high lightpath setup/teardown rate of $1 \mathrm{MHz}$ was achieved by spatiotemporal synchronization network control [15].

\subsection{Low-latency metro-access optical networks}

The requirement of ms-level end-to-end (E2E) latency for $5 \mathrm{G}$ real-time applications poses a huge challenge to 
the bearer networks. Optically bypassing the IP routers using all-optical transmission and switching technologies becomes a potential solution. However, current optical access networks adopting the passive optical network (PON) technologies can not support cross-PON E2E lightpath establishment due to the lack of cross-PON coordination mechanism, presenting a significant obstacle for the realization of low latency in large-scale networks. To solve this problem, a new metro-access converged network architecture was proposed based on inter-PON time synchronization [16]. By enabling coordinated time slot assignment among PONs, cross-PON lightpaths can be established to reduce E2E latency by almost two orders of magnitude.

\subsection{Large-scale smart-grid communication networks}

The optical network is regarded as a promising candidate for smart grid communications due to its unique advantages such as low latency and jitter. However, there exists a significant gap between the number of lightpaths required for the latency-sensitive traffic in large-scale smart grid, e.g. teleprotection and under frequency load shedding (UFLS), and that can be offered by optical networks at the wavelength granularity. Luckily, the optical time slice switching (OTSS) technology based on spatiotemporal synchronization is able to offer over one thousand sub-channels on a single wavelength channel, and thus can become a promising solution [17]. Numerical results showed that by using OTSS, the E2E latencies of the latency-sensitive traffic can be reduced by $60 \%$ under heavy load.

\section{CONCLUSION}

The emerging real-time applications place new requirements in the dynamic performance of optical networks. Because of the uncertainty of signaling transmission delay, the traditional "message-triggered" control mechanism becomes a major bottleneck to network dynamics. In order to improve the dynamic performance of optical networks, we introduce a novel spatiotemporal synchronization optical network control architecture which is enabled by high-precision time synchronization, and its typical application scenarios as well. Preliminary results show that by establishing control coordination among nodes and controllers, the proposed architecture can improve the network dynamic performance by orders of magnitude.

\section{ACKNOWLEDGMENTS}

This work was supported in part by National Key R\&D Program of China under grant No. 2018YFB1801702, and NSFC under grants No. 61871448 and 61621064.

\section{REFERENCES}

[1] C. F. Lam, et al., "Fiber optic communication technologies: What's needed for datacenter network operations," Communications Magazine, 2010, 48(7).

[2] M. A. Taubenblatt, "Optical interconnects for high-performance computing," Journal of Lightwave Technology, 2012, 30(4): 448-457.

[3] "5G Vision and Requirements," IMT-2020 White Paper.

[4] "Architecture for the automatically switched optical network (ASON)," ITU-T R. G. 8080/Y. 1304, 2001.

[5] W. Liu, et al., "Intelligent inter-domain connection provisioning for multi-domain multi-vendor optical networks," Journal of Optical Communications and Networking, 2015, 7(3): 176-192.

[6] R. Jing, et al., "Field trial of virtual transport network services based on hierarchical control over multi-domain OTN networks", in Proc. Optoelectronics \& Communications Conference, TuA1-2, 2016.

[7] L. Han, et al., "First national high-precision time synchronization network with sub-microsecond accuracy over commercial optical networks for wireless applications," in Proc. Asia Communications and Photonics Conference, AF4B.6, 2014.

[8] L. Han, et al., "A distributed time synchronization solution without satellite time reference for mobile communication," IEEE Communications Letters, 2013, 17(7): 1447-1450.

[9] R. Luo, et al., "High-reliability sub-nanosecond network time synchronization method enabled by double-frequency distributed time synchronization," Journal of Optical Communications and Networking, 2019, 11(1): A40-A51.

[10] N. Hua, et al., "Optical time slice switching (OTSS): an all-optical sub-wavelength solution based on time synchronization," in Proc. Asia Communications and Photonics Conference, AW3H.3, 2013.

[11] N. Hua, et al., "All-optical time slice switching method and system based on time synchronization," U.S. Patent, 9608763, 2017.

[12] Y. Li, et al., "Fine-grained all-optical switching based on optical time slice switching for hybrid packet-OCS intra-data center networks", in Proc. Optical Fiber Communication Conference, W3J.5, 2016.

[13] R. Luo, et al., "Achieving ultralow-latency optical interconnection for high performance computing (HPC) systems by joint allocation of computation and communication resources," in Proc. Optical Fiber Communication Conference, W1J.4, 2019.

[14] R. Luo, et al., "Fast parallel lightpath re-optimization for space-division multiplexing optical networks based on time synchronization," Journal of Optical Communications and Networking, 2018, 10(1): A8-A19.

[15] Y. Li, et al., "Fast lightpath hopping enabled by time synchronization for optical network security," IEEE Communications Letters, 2015, 20(1), 101-104.

[16] J. Li, et al., "A flexible low-latency metro-access converged network approach based on timesynchronized TWDM-PON," in Proc. Optical Fiber Communication Conference, Th2A.50, 2018.

[17] Z. Zhong, et al., "Evolving optical networks for latency-sensitive smart-grid communications via optical time slice switching (OTSS) technologies," in Proc. Optoelectronics \& Communications Conference, 1st Place Poster Award, 2017. 